\title{
Globe
}

Revue internationale d'études québécoises

\section{Lise Gauvin : Langagement. L'écrivain et la langue au Québec. Montréal, Boréal, 2000}

\section{John Kristian Sanaker}

Volume 4, numéro 1, 2001

URI : https://id.erudit.org/iderudit/1000608ar

DOI : https://doi.org/10.7202/1000608ar

Aller au sommaire du numéro

Éditeur(s)

Globe, Revue internationale d'études québécoises

ISSN

1481-5869 (imprimé)

1923-8231 (numérique)

Découvrir la revue

Citer ce compte rendu

Sanaker, J. K. (2001). Compte rendu de [Lise Gauvin : Langagement. L'écrivain et la langue au Québec. Montréal, Boréal, 2000]. Globe, 4(1), 150-152.

https://doi.org/10.7202/1000608ar d'utilisation que vous pouvez consulter en ligne.

https://apropos.erudit.org/fr/usagers/politique-dutilisation/ 


\section{REVUE INTERNATIONALE D'ÉTUDES QUÉBÉCOISES}

\section{Lise Gauvin}

Langagement. L'écrivain et la langue au Québec. Montréal, Boréal, 2000.

Les travaux de Lise Gauvin sont marqués par sa double appartenance professionnelle. Professeure de littérature française à l'Université de Montréal depuis de nombreuses années, elle connaît aussi une carrière d'écrivaine (nouvelles et autres textes de fiction) ; combinaison qui fait d'elle une observatrice perspicace de la situation d'écriture des autres (voir aussi ses nombreux entretiens avec des écrivains francophones).

Aussi une bonne partie de sa recherche la plus récente porte-t-elle sur les rapports qu'entretiennent les écrivains avec leur propre langue. Lise Gauvin s'est surtout penchée sur la situation des écrivains francophones (québécois, antillais, etc.), situation qu'elle a proposé de circonscrire en utilisant le terme de surconscience linguistique : il s'agit d'une "conscience aiguë de la langue comme objet de réflexion, d'interrogation, d'enquête mais aussi de transformation et de création * (p. 209). Cette nécessité de réfléchir sur la langue est une obligation à laquelle l'écrivain francophone ne peut pas se dérober, vu sa situation de contact avec l'(les) autre(s) culture(s) ainsi que son besoin de se définir par rapport à la France et à la culture "mère ". L'écrivain francophone est "condamné à penser la langue", dit Gauvin (p. 9).

Langagement se présente comme un travail de synthèse sur la problématique de la surconscience linguistique dans une optique québécoise. Gauvin y reprend certains des sujets et des écrivains étudiés dans des travaux antérieurs (Crémazie, Poulin, Ducharme, etc.), tout en ajoutant du nouveau. Même ceux qui connaissent bien la position de Gauvin dans ce domaine auraient intérêt à lire Langagement, car l'ouvrage ajoute une dimension diachronique intéressante aux études ponctuelles antérieures.

L'étude comporte deux parties distinctes: une première partie où Gauvin présente la diversité des prises de position théoriques devant la question de la langue de l'expression littéraire, une deuxième qui est 
consacrée à des écrivains qui font preuve de "surconscience linguistique "dans leur pratique fictionnelle. La partie théorique comporte des chapitres sur Crémazie et sa réflexion sur la nécessité d'avoir une "langue à soi " (rêve et mythe tenaces qui se manifestent une dernière fois chez les partisans du joual), sur les prises de position des écrivains de Parti pris, sur les nombreux manifestes qui entretiennent des rapports intimes avec l'actualité culturelle et politique, et finalement un chapitre très riche qui présente le foisonnement de la pensée féministe des années 1970.

Dans les chapitres qui abordent les diverses pratiques textuelles, Gauvin montre qu'il existe une évolution nette d'un réalisme "sage " qui distingue nettement la langue "correcte " du narrateur du discours "marqué " des personnages, vers une conception de la littérature où le texte entier est davantage une "parole spontanée "(p. 141, à propos de Tremblay). Un premier chapitre est consacré à la tradition réaliste représentée entre autres par les romans "canoniques" que sont Bonbeur d'occasion et Le survenant; Gauvin nous propose ensuite une analyse très concrète et précise de la langue de Michel Tremblay qui, dans l'optique de Gauvin, assure une "normalisation " (et donc une transformation littéraire) du langage populaire au lieu d'en être le reflet; un chapitre est voué au groupe très hétérogène constitué par Yves Beauchemin, Yolande Villemaire, Jacques Poulin, Francine Noël et Gérald Tougas, chez qui on observe, cependant, un trait commun important, à savoir "une tolérance toute particulière au plurilinguisme " (p. 164); le chapitre sur la langue romanesque de Réjean Ducharme montre à l'évidence combien cette écriture est complexe - et aussi dans quelle mesure elle requiert une compétence linguistique très poussée chez le lecteur; et finalement "L'écriture nomade "nous introduit à la riche tradition des écrivains "venus d'ailleurs ", représentés par Régine Robin (née à Paris de parents d'origine juive polonaise), Emile Ollivier (qui quitte Haïti à vingt-cinq ans), Marco Micone (qui arrive à Montréal d'Italie à l'âge de treize ans) et Ying Chen (immigrée de Shangai dans les années 1980). Si Gauvin a choisi de terminer son parcours de l'histoire de la surconscience linguistique de l'écrivain québécois par cette série de portraits d'écrivains néo-québécois, c'est sans doute parce qu'ils représentent en quelque sorte un redoublement de la 
surconscience linguistique dans la mesure où ils ajoutent à leur statut de francophones décentrés leur expérience de passage d'une langue maternelle autre à une nouvelle langue d'expression.

En lisant Langagement, on a tout le temps le sentiment rassurant d'être bien guidé, non seulement par une spécialiste qui parle d'une chose qu'elle connaît à fond, mais qui prend aussi plaisir à le faire. Bref, on devine chez Lise Gauvin une passion et un enthousiasme qu'elle réussit à transmettre à ses lecteurs.

Le plaisir qu'il y a à lire Langagement est aussi dû à l'efficacité de sa méthode. Gauvin ne fait pas une analyse formaliste et rigide où tous les auteurs sont lus à travers la même grille de lecture. Elle propose plutôt des repères de lecture très souples qui lui permettent de mettre en valeur la particularité de chaque écrivain et de chaque période. On a la nette impression que sa lecture fait valoir les différences, sans réduire la spécificité des textes analysés. En outre, vouloir présenter Crémazie et Régine Robert dans la même optique et dans le même ouvrage, sans réduire leur spécificité et ce, tout en montrant ce qu'ils ont en commun, cela ne va pas de soi.

John Kristian Sanaker Université de Bergen (Norvège)

\section{Kenneth McRoberts}

Un pays à refaire.

Montréal, Éditions du Boréal, 1999.

Kenneth McRoberts est l'un des auteurs canadiens bien connus au Québec. Ses thèses récentes, développées dans Misconceiving Canada. The Struggle for National Unity puis traduites et publiées aux Éditions du Boréal, rejoindront sûrement les lecteurs qui rêvent, comme lui, de refaire le Canada. Avec elles, on apprend que son pays, le Canada, est en crise accélérée depuis le passage de Pierre Elliott Trudeau à la tête du gouvernement canadien. Et, pour en sortir, il faudrait faire un retour 